\title{
Cadaveric findings of the effect of rib fractures on spread of serratus plane injections
}

\author{
David F. Johnston, MB, BCh, BAO, FRCA, EDRA (1) - Nicholas D. Black, MB, BCh, \\ BAO, FRCA · Rachael O'Halloran · Lloyd R. Turbitt, MB, BCh, BAO, FRCA, MSc • \\ Samantha J. Taylor, BMedSc (Hons), MPhil, EdD
}

Received: 16 October 2018/Revised: 5 February 2019/Accepted: 11 February 2019/Published online: 7 March 2019

(C) Canadian Anesthesiologists' Society 2019

\section{To the Editor,}

Serratus plane (SP) blocks provide effective analgesia for patients with rib fractures following chest trauma and are an attractive alternative if thoracic epidural analgesia is contra-indicated. ${ }^{1}$ Serratus plane blocks involve injecting local anesthetic superficial to the serratus anterior muscle (SAM) in the mid-axillary line using ultrasound guidance. ${ }^{2}$ A cadaveric study showed spread of SP injectate to be limited to the lateral cutaneous branches of intercostal nerves with no direct spread between fascial layers deep enough to reach the intercostal nerves. ${ }^{3}$

Rib periosteum and intercostal muscles are, however, innervated by the intercostal nerves, which travel between internal and innermost intercostal muscles. Thus, effective analgesia for rib fractures logically requires local anesthetic to spread across multiple tissue planes to affect this deeper layer. We wanted to show "If the presence of rib fractures facilitated spread of SP injectate towards deeper structures?"

D. F. Johnston, MB, BCh, BAO, FRCA, EDRA ( $\square)$.

L. R. Turbitt, MB, BCh, BAO, FRCA, MSc

Royal Victoria Hospital, Belfast Health \& Social Care Trust,

Belfast, UK

e-mail: davidf.johnston@belfasttrust.hscni.net

N. D. Black, MB, BCh, BAO, FRCA

Toronto Western Hospital, Toronto, ON, Canada

R. O'Halloran

Queen's University Belfast, Belfast, UK

S. J. Taylor, BMedSc (Hons), MPhil, EdD

Centre for Biomedical Sciences Education, Queen's University

Belfast, Belfast, UK
We performed SP injections in two thawed fresh frozen cadavers, which acted as their own controls. Ethical approval was granted by the Queen's University, Belfast School of Medicine, Dentistry and Biomedical Sciences Research Ethics Committee (ref. 18.35v2). One hemithorax underwent four rib fractures (from ribs 2-6) using manual digital pressure at ultrasound-guided predetermined locations in the mid-clavicular line. The other hemithorax acted as a control with no rib fractures. Bilateral SP injections were performed using $30 \mathrm{~mL}$ of ink and methylcellulose in the mid-axillary line at the fourth intercostal space with the needle tip placed in the interfascial plane deep to SAM at the anterior edge of latisimus dorsi muscle. After $30 \mathrm{~min}$, cadavers were dissected using a midline incision and medial-to-lateral incisions at the clavicle and inferior costal margins, which extended to the posterior axillary line. Muscle layers were sequentially reflected laterally with close observation of superior-inferior, medial-lateral-posterior according to anatomical lines and superficial-deep spread according to muscle layers.

Dissections of the hemithorax with rib fractures revealed consistent spread of injectate to multiple intercostal nerves and surrounding deep intercostal muscle layers (Figure a). In the control hemithoraces, spread was contained to within the SP with no staining of the external intercostal muscle fascia or rib surfaces (Figure b). More posterior spread towards the medial border of the scapula was observed in both rib fracture hemithoraces but was limited to the anterior border of the latissimus dorsi muscle on the nontraumatized hemithoraces.

The cadaveric findings support the theory that disruption of tissue planes from trauma may actually facilitate deeper spread of SP injectate to affect the intercostal nerves. Furthermore, it is conceivable that injectate could extend deep to iliocostalis and longissimus thoracis muscles based on the finding of further posterior extent of SP spread in the 


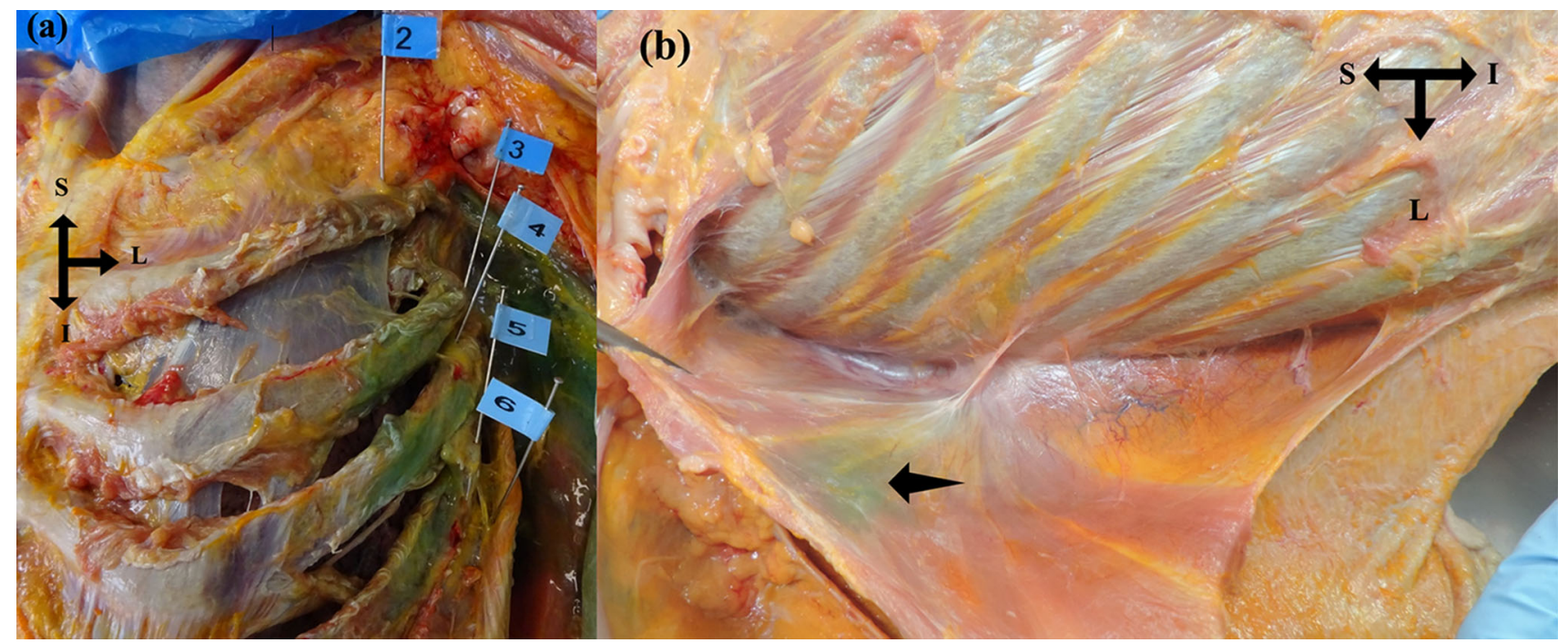

Figure a) Hemithorax with rib fractures. Blue numbered markers show exact location of rib fractures on the respective ribs. b) Nonfractured hemithorax demonstrating absence of dye penetration deeper than the serratus anterior muscle and absence of staining of superficial fascia of external intercostal muscles. Black arrow showing containment of dye superficial to serratus muscle. $\mathrm{I}=$ inferior; $\mathrm{L}=$ lateral; $\mathrm{S}=$ superior fractured specimens. From here, one could expect diffusion anteriorly into the paravertebral space, thus conferring a visceral analgesic component from SP blocks. This "continuous plane" has been shown by the newly described "rhomboid intercostal and subserratus plane" block. ${ }^{4}$

In cadavers, the presence of rib fractures facilitated a deeper and more posterior spread of SP injections when compared with a hemithorax with intact ribs.

Competing interests No external funding and no competing interests declared

Editorial responsibility This submission was handled by Dr. Gregory L. Bryson, Deputy Editor-in-Chief, Canadian Journal of Anesthesia.

\section{References}

1. Kunhabdulla NP, Agarwal A, Gaur A, Gautam SK, Gupta R, Agarwal A. Serratus anterior plane block for multiple rib fractures. Pain Physician 2014; 17: E553-5.

2. Blanco R, Parras T, McDonnell JG, Prats-Galino A. Serratus plane block: a novel ultrasound-guided thoracic nerve wall block. Anaesthesia 2013; 68: 1107-13.

3. Mayes J, Davison E, Panahi P, et al. An anatomical evaluation of the serratus anterior plane block. Anesthesia 2016; 71: 1064-9.

4. Elsharkawy H, Maniker R, Bolash R, Kalasbail P, Drake RL, Elkassabany $N$. Rhomboid intercostal and subserratus plane block a cadaveric and clinical evaluation. Reg Anesth Pain Med 2018; 43: 745-51.

Publisher's Note Springer Nature remains neutral with regard to jurisdictional claims in published maps and institutional affiliations. 